\title{
Cardiac Ventricle
}

National Cancer Institute

\section{Source}

National Cancer Institute. Cardiac Ventricle. NCI Thesaurus. Code C12730.

One of the two lower chambers of the heart that receives blood from an atrium and pumps blood to the arteries. 